\title{
El trabajo en equipo en la formación inicial del profesorado
}

\author{
Teamwork in initial teacher training \\ José Luis Aparicio-Herguedas, Carlos Velázquez-Callado, Antonio Fraile-Aranda
}

Facultad de Educación y Trabajo Social. Universidad de Valladolid. España.

\section{CORRESPONDENCIA:}

José Luis Aparicio Herguedas

joseluis.aparicio.herguedas@uva.es

Recepción: agosto 2020 • Aceptación: marzo 2021
CÓMO CITAR EL ARTÍCULO:

Aparicio-Herguedas, J.L., Velázquez-Callado, C., \& Fraile-Aranda, A. (2021). El trabajo en equipo en la formación inicial del profesorado. Cultura, Ciencia y Deporte, 16(49), 455-464. http://dx.doi. org/10.12800/ccd.v16i49.1548

\section{Resumen}

El desarrollo competencial en la formación del profesorado es una temática especialmente relevante en el ámbito de la educación superior. La competencia para el trabajo en equipo es valiosamente considerada en el sector educativo profesional e imprescindible para desarrollar la innovación educativa en los centros. En el presente trabajo se analiza cómo se aplican las dinámicas de trabajo en equipo en la formación inicial del profesorado de educación física y si estas permiten el desarrollo de esta competencia. Para dicho estudio se valora la percepción de un grupo de diez estudiantes de los Grados de Maestro en Educación Primaria (Educación Física) y de Ciencias de la Actividad Física y del Deporte sobre las dinámicas y experiencias de trabajo en equipo realizadas durante su formación. El estudio adopta una perspectiva metodológica cualitativa, a partir del análisis de los datos procedentes de un grupo de discusión, mediante el software ATLAS.ti. Tras el proceso analítico de codificación y categorización se obtienen resultados que dibujan un modelo formativo basado en el trabajo en grupo que no capacita suficientemente para la adquisición de esta competencia. No obstante, los estudiantes muestran una actitud prosocial de base imprescindible para afrontar los retos de la cooperación.

Palabras clave: trabajo en equipo, aprendizaje cooperativo, competencias docentes, evaluación formativa, proceso de enseñanza-aprendizaje.

\begin{abstract}
Competency development in Initial Teacher Training is a particularly relevant issue in the field of higher education. Teamwork Competence is considered valuable in the professional education sector and is essential for developing educational innovation at schools. This paper analyzes how teamwork dynamics are applied in the initial teacher training of future physical education teachers and whether they allow the development of this competence. For this, the perception of ten students of the Primary Education (Physical Education) and Sciences of Physical Activity and Sport degrees about the dynamics and experiences of teamwork carried out during their education are examined. The study adopts a qualitative methodological perspective, based on the analysis of the data collected from a discussion group. Through ATLAS.ti software, information was coded and categorized. After this analytical process, results draw a formative model based on teamwork that does not sufficiently qualify for the acquisition of this competence. However, the students show a basic prosocial attitude which is essential to face the challenges of cooperation.
\end{abstract}

Key words: teamwork, cooperative learning, teaching competencies, formative assessment, teaching-learning process. 


\section{Introducción}

El trabajo en equipo es una de las competencias transversales más valoradas en el sector educativo y una de las estrategias docentes en la formación inicial del profesorado, con un impacto directo en el futuro comportamiento profesional de los docentes y con aplicabilidad a los cambios sociales (Ní Chróinín \& O'Sullivan, 2014). Diversas investigaciones manifiestan la importancia de integrar en las organizaciones profesionales diversos perfiles con habilidades para trabajar en equipo, reconociendo que esta competencia multidimensional es de vital importancia por su efectividad a la hora de abordar las tareas, alcanzar las metas establecidas y resolver problemas (Cano \& Fernández, 2016; Chen et al., 2004; Leach et al., 2005; Torrelles et al., 2011; Torrelles et al., 2015).

Dentro del sector educativo, en el esfuerzo de adaptación a una sociedad cambiante (Molina \& Antolín, 2008), la participación interdisciplinar e interprofesional para desarrollar proyectos, resolver problemáticas, interrelacionar conocimientos, atender las necesidades del alumnado, adaptar el currículo, incorporar las competencias básicas a los programas de aula, requieren superar la individualidad y actuar de forma colegiada cooperando (Méndez-Alonso, 2016; Villardón-Gallego, 2015). A pesar de los estudios existentes sobre trabajo en equipo, las dimensiones que lo conforman, las dinámicas formativas para desarrollarlo o la gestión de la evaluación durante su práctica (Fraile et al., 2013; Howe, 2011; Pérez-Pueyo et al., 2013; Prieto et al., 2018) no existen apenas investigaciones que muestren si el esfuerzo formativo realizado en la formación del profesorado favorece la adquisición de esta competencia. Se considera por ello importante, en el contexto de la educación superior, conocer la percepción de los estudiantes, en cuanto participantes de primera mano, en las diversas dinámicas para aprender a trabajar en equipo realizadas durante su formación.

En la educación superior, las metodologías basadas en el trabajo grupal están ampliamente extendidas, de forma que se constata la urgencia en el contexto universitario de "trabajar juntos", siendo el grupo el punto de partida para desarrollar aspectos competenciales como la toma de decisiones, el consenso y la resolución de problemas (Moreno-Andrés et al., 2010; Scallon, 2004; Vallejo-Ruiz \& Molina-Saorín, 2011), todo desde un enfoque formativo en el que es habitual valorar el contenido a desarrollar, en detrimento del proceso de desarrollo del equipo, que es donde radica la esencia del aprendizaje y la adquisición de la competencia (Asún et al., 2019).
A nivel epistemológico se parte de la falta de acuerdo en cuanto a terminología sobre lo que es el trabajo en equipo, en relación a otros conceptos afines que subyacen en los discursos que lo definen y explican: trabajo en grupo, grupos de trabajo, trabajo en equipo o equipos de trabajo (Torrelles et al., 2014). Así, un equipo se define como la unión de dos o más personas que trabajan en cooperación, creando una red de relaciones de interdependencia, estableciendo entre ellas una sinergia y cohesión con el propósito de alcanzar una meta preestablecida para el bien de su organización de pertenencia y adaptándose a las particularidades del contexto (Kozlowski \& Ilgen, 2006; Ros, 2006; Torrelles et al., 2014). Los equipos se asientan sobre tres niveles de ordenación, que hacen que el trabajo compartido sea eficaz: la organización, el equipo y la persona, siendo este último el nivel más importante.

En el equipo, la persona habilita sus conocimientos, capacidades y habilidades para el fin común, formándose para contribuir a su liderazgo. Ros (2006) considera que se trata de un estilo para la realización de una actividad académica o laboral, en la que el individuo interacciona con los demás inspirando un nuevo espíritu de participación conjunta, cooperando, comunicándose, confiando, apoyándose mutuamente y considerando responsablemente la misión del equipo como propia. Siguiendo a Cannon-Bowers et al. (1995), trabajar en equipo incluye el conocimiento de las bases conceptuales y procedimentales para la actuación eficaz del equipo en la gestión de la tarea, junto con el comportamiento en las relaciones interpersonales, a fin de promover una dinámica común de trabajo, responsabilizándose recíprocamente de las tareas y del abordaje de las adversidades.

Villa y Poblete (2007) consideran esencial el desarrollo de la responsabilidad personal entre los miembros de los equipos. En un primer momento, comprometiéndose formalmente en la realización de las tareas, cumpliendo los plazos y asumiendo los objetivos grupales frente a los intereses individuales. Después, trascendiendo la mera participación formal, incentivando también la comunicación, el entendimiento y la armonía entre los componentes.

En el ámbito formativo, se atribuyen al docente las tareas de organización, dirección y supervisión del proceso de trabajo en equipo. Él es quien ha de velar por el cumplimiento de los principios de aprendizaje cooperativo, reforzar el proceso de trabajo conjunto sobre el resultado final, atribuir funciones a sus miembros y especialmente promover un modelo de evaluación formativa durante todo el proceso de aprendizaje de los estudiantes (Zapatero et al., 2018), por ejemplo, aplicando estrategias de autoevaluación y coevaluación que faciliten la corresponsabilidad evaluadora entre el 
alumnado (Méndez-Giménez \& Fernández-Río, 2013; Velázquez et al., 2014; Velázquez, 2013). En este sentido, Pérez-Pueyo, et al. (2020) consideran la evaluación formativa como una herramienta indispensable para conectar a los estudiantes con la aplicabilidad real de la cooperación, buscando que los aprendizajes sean auténticos. La autoevalución y la coevaluación son estrategias que permiten la interiorización de los principios de la cooperación y el trabajo en equipo desde su máxima implicación (López-Pastor \& Pérez-Pueyo, 2017).

Ocasionalmente, los estudiantes que participan en torno a metodologías de trabajo grupal atraviesan situaciones críticas que ponen en peligro el proceso, la tarea a desarrollar y la propia vida del equipo, pues las conductas desatadas tienden más hacia la conflictividad que a la cooperación (Cuadrado et al., 2012; Del Canto et al., 2009; Jhen \& Mannix, 2017). Los mismos autores describen situaciones de conflicto frecuentes, a partir de las diferencias entre las capacidades individuales de los miembros del equipo y sus aportaciones. Entre ellas, se destacan renuncias en la realización de algunas tareas, deficiente gestión de los conflictos y malestar cuando la calificación es idéntica para todos sus miembros, considerando que todos no han participado de manera corresponsable y equitativa en la realización de las tareas.

Igualmente, con estudiantes de magisterio, Aguilera (2018) destaca las dificultades que estos experimentan para las tareas grupales, a partir de un esfuerzo desigual al desarrollar dichas tareas, debido al excesivo número de participantes. Asimismo, en experiencias de trabajo en equipo en la formación del profesorado de Educación Física, se describieron situaciones de conflicto que acabaron derivando más en el individualismo, la competitividad y la insuficiencia comunicativa que en la colaboración (Fraile, 2008; Fraile et al., 2009).

Salas et al. (2005) plantean que el desarrollo de la competencia para el trabajo en equipo requiere del tratamiento de varios componentes que facilitan la efectividad del equipo: el liderazgo compartido, el seguimiento mutuo del desarrollo de la tarea, el comportamiento de respaldo recíproco, así como la adaptabilidad y la orientación como equipo; además, los miembros del equipo han de compartir los mismos modelos mentales (creencias compatibles), desarrollar la confianza mutua y comunicarse entre sí. En la formación del profesorado, el trabajo de estos aspectos toma sentido si se plantean y desarrollan cooperativamente actividades de resolución de problemas, de forma que aseguren la utilidad, la efectividad y la significatividad de la tarea (Gámez \& Torres, 2013; Sáiz \& Gómez, 2011; Torrelles et al., 2011). Por ello, se plantea el Aprendizaje Cooperativo como estrategia metodológica que facilita el desarrollo de estos componentes y, en consecuencia, la transición desde el mero trabajo grupal al trabajo en equipo orientado a generar aprendizajes. En este sentido, Johnson y Johnson (1999) destacan la necesidad de promover la presencia de cinco elementos esenciales para orientar eficazmente las experiencias prácticas de trabajo grupal: interdependencia positiva de metas, interacción promotora, responsabilidad individual, habilidades interpersonales y autoevaluación grupal del proceso y de los resultados obtenidos. Desde estas premisas, el Aprendizaje Cooperativo se presenta, por tanto, como una metodología que estructura el trabajo grupal con el objetivo de ayudar a superar los problemas de los equipos, facilitando que los estudiantes aprendan que las tareas no son el fin sino el medio, alcancen los objetivos académicos y también sociales, interaccionen unos con otros en grupos reducidos y heterogéneos, se comuniquen, a fin de lograr un cambio en su comportamiento y en la forma de relacionarse, preocupándose responsablemente unos por otros, movilizando para ello diversos conocimientos, habilidades y actitudes (Ferriz-Valero, et al., 2019; Velázquez, 2013). Ahora bien, la implementación del Aprendizaje Cooperativo no es una tarea sencilla, requiere de mucho tiempo y de un arduo trabajo por parte del docente (Casey \& Dyson, 2009). En este sentido, algunos autores plantean un proceso estructurado que implica acciones, a diferentes niveles, para generar cohesión grupal y para promover las habilidades necesarias que permitan a los estudiantes gestionar las relaciones interpersonales y la comunicación, imprescindibles para aprender a cooperar (Fernández-Río, 2017; Gallego-Ortega \& Rodríguez-Fuentes, 2018; Velázquez, 2018). En definitiva, el docente debe facilitar la transición de los grupos en equipos que manejen habilidades y actitudes de comunicación interpersonal, de negociación, de cesión, de ayuda mutua, de asunción de responsabilidades y de resolución de problemas, para generar aprendizajes en todos y cada uno de los miembros del equipo. Así, y en virtud de lo expuesto, en este estudio se pretende averiguar cómo se desarrollan las dinámicas de trabajo en equipo en la formación inicial del profesorado de educación física y si estas facilitan situaciones de verdadero aprendizaje de esta competencia.

\section{Metodología}

\section{Diseño}

La metodología de investigación es de carácter cualitativo. Según Yilmaz (2013) permite comprender a las personas, los fenómenos, los acontecimientos y las 
situaciones sociales en sus entornos y procesos naturales desde un planteamiento interpretativo, donde se revelan los posibles significados del fenómeno y desde la voz de las personas implicadas o, como plantean Fossey et al. (2002), al contribuir a la compresión de los significados de las vidas de los participantes y su cosmovisión del mundo. En este sentido, se escucha y observa a los estudiantes de educación física, que en su formación inicial han vivenciado múltiples formas y tipos de prácticas de trabajo grupal, de modo que su perspectiva se considera de máximo valor para comprender este fenómeno.

\section{Participantes}

La muestra la conforman 10 estudiantes procedentes de diversas universidades españolas. De ellos, 5 cursan cuarto curso del Grado Universitario de Ciencias de la Actividad Física y el Deporte (CAFyD) y 5 lo hacen en cuarto curso del Grado Universitario de Maestro de Educación Primaria con la mención en Educación Física (MEF). Las edades de los 10 estudiantes oscilan entre los 22 y 24 años. 5 son hombres y 5 son mujeres.

\section{Instrumentos y procedimiento de actuación}

Los datos se obtuvieron en el curso 2018 a partir de un grupo de discusión con los 10 estudiantes mencionados, representantes de las dos titulaciones de Educación Física vigentes actualmente (CAFyD y MEF). Tal y como establecen Bloor et al. (2001), se comprueba que los miembros del grupo comparten características importantes para la discusión, como son: el interés por el tema, la especialización cursada, los intereses profesionales y el momento formativo final en el que se encuentran, próximo al mercado laboral. A través de la discusión grupal, cada uno de los participantes pudo exponer, desde su percepción, la influencia de las dinámicas de trabajo grupal desarrolladas durante las asignaturas cursadas, y si estas contribuyeron al trabajo en equipo. El grupo de discusión permitió conocer qué piensan los estudiantes y por qué lo hacen, mostrando los problemas y las preocupaciones de manera fundamentada, expresando su conocimiento y experiencia en relación al fenómeno objeto de estudio (Barbour, 2014; Barbour \& Kitzinger, 1999).

La dinamización del grupo de discusión se desarrolló a partir de varias preguntas orientativas a fin de optimizar las intervenciones de los participantes.

a) ¿Qué valor tiene la competencia para trabajar en equipo, a la luz de la formación cursada y de cara al futuro profesional como docentes de educación física? b) ¿Qué actividades formativas han ayudado a la adquisición de la competencia para trabajar en equipo durante las asignaturas cursadas?

c) ¿Cuál es el funcionamiento de las experiencias de trabajo en equipo que se han desarrollado durante la formación cursada, para el desarrollo de la competencia para el trabajo en equipo?

d) ¿De qué forma la evaluación formativa, si se hubiera dado, ha favorecido la adquisición de la competencia para trabajar en equipo?

e) ¿Qué procedimientos de evaluación se han utilizado para valorar las dinámicas de trabajo en equipo y los consiguientes productos finales?

f) ¿Existe alguna transferencia de los aprendizajes realizados desde las experiencias de trabajo en equipo, desarrolladas en las asignaturas cursadas, con la formación práctica realizada en los centros educativos?

\section{Análisis de datos}

Se utilizó el software ATLAS.ti para analizar los datos cualitativos procedentes de la transcripción del grupo de discusión a partir de dos ciclos de codificación (Miles et al., 2014). Durante el primer ciclo se analizaron los datos detenida y reflexivamente, obteniendo códigos que dimensionaban y caracterizaban las dinámicas de trabajo en equipo realizadas por los estudiantes en clase.

Se aplicaron diversos enfoques de codificación (Saldaña, 2009): descriptivo, in vivo, emocionales, de proceso y de atributo. Durante el segundo ciclo, se realizó una codificación de tipo axial precisando, a partir de los códigos obtenidos, nuevas agrupaciones, conformando nuevos códigos (Gibbs, 2012; Strauss \& Corbin, 1998) (tabla 1).

Se consideró alcanzada la saturación en el momento en el que las comparaciones entre los datos dejaron de evidenciar nuevas relaciones y propiedades entre ellos. Así se dio por agotada la riqueza representativa de los datos en un proceso analítico de carácter circular (Flick, 2007).

Los códigos resultantes fueron analizados de forma crítica por los tres investigadores participantes en el estudio, mediante un debate reflexivo sobre su conceptualización en un proceso de triangulación en base a las fuentes.

Este debate facilitó la regeneración, perfeccionamiento y refinamiento de los códigos emergentes, favoreciendo la fiabilidad, la credibilidad y la transferencia del estudio (Guba, 1985; Watling Neal et al., 2015; Wolf \& Silver, 2017). Los códigos y definiciones operacionales finales fueron los siguientes (tabla 2). 
Tabla 1. Resultado del proceso de codificación.

\begin{tabular}{lc}
\hline Códigos de primer ciclo & Codificación axial \\
\hline Aprendizaje por repetición & Formación \\
Trabajo grupal continuado & \\
Dinámicas repetidas & Procedimiento \\
Reconociendo errores & \\
Aprendizaje autodidacta & \\
Nadie te enseña & \\
\hline Repartiendo los epígrafes del trabajo & \\
Repartiendo y realizando tareas & \\
Dialogando & \\
Asistiendo a reuniones & \\
Trabajando en drive & \\
\hline
\end{tabular}

Evaluar solo contenidos

Prevalencia de contenido

Atomización de los contenidos de estudio

Prevalencia del documento final y su contenido

"Injusta valoración final de contenidos y no de proceso"

"Lamentable cuántas personas tan irresponsables"

Conflicto de responsabilidades

Trabajando en grupo se trabaja el doble

Disconformidad en la exigencia de resultados

Diferencia de intereses

Ausencia de confianza y respeto

Trabajo grupal despreciado

Prevalencia del producto final

Evaluación centrada en resultado

Valoración del resultado frente al proceso

Calificaciones injustas solo desde resultados

Profesor no ve más que el resultado

Presión del tiempo para entregas

Necesidad de seguimiento del proceso

Uso de seminarios grupales

Uso del diario del profesor

Evaluar conjuntamente el profesor y el grupo

Evaluar el grupo

Consensuar la calificación

Verificar que todos saben

Supervisando el trabajo de los grupos

Monitoreo del profesor

Observando quien trabaja en los grupos en el aula

Saber quien colabora

Observando la participación de los miembros del grupo

Preguntando al grupo y sus miembros

Mediador en conflictos

Ayudando a resolver problemas

Tabla 2. Definición de los códigos finales.

\begin{tabular}{ll}
\hline Código & Definición operacional \\
\hline Formación & Aprendizajes realizados sobre el trabajo en equipo. \\
\hline Procedimiento & $\begin{array}{l}\text { Caracterización del método aplicado para trabajar en equipo en las dinámicas formativas } \\
\text { establecidas. }\end{array}$ \\
\hline Prevalencia de contenido & Presencia del contenido curricular a aprender en las tareas en equipo a realizar. \\
\hline Conflicto de responsabilidades & $\begin{array}{l}\text { Tipificación de la conflictividad desatada en el seno de los equipos de trabajo durante las } \\
\text { dinámicas formativas desarrolladas. }\end{array}$ \\
\hline Evaluación centrada en el resultado & $\begin{array}{l}\text { Proceso de evaluación experimentado en las dinámicas formativas de trabajo en equipo y su } \\
\text { caracterización. }\end{array}$ \\
\hline Evaluación demandada & $\begin{array}{l}\text { Preferencia de evaluación de los estudiantes para el desarrollo de la competencia para trabajar } \\
\text { en equipo. }\end{array}$ \\
\hline Monitoreo del profesorado & Rol del docente en la gestión de las dinámicas del trabajo en equipo. \\
\hline
\end{tabular}




\section{Resultados}

Tras los resultados obtenidos del grupo de discusión y las dinámicas del trabajo en equipo, en ambas titulaciones, se presentan 96 evidencias. Los códigos establecidos junto a su enraizamiento (frecuencia), fueron los siguientes: formación (16); procedimiento; (16) evaluación demandada (15); monitoreo del profesor (14); conflicto de responsabilidades (12); prevalencia del contenido (11) y evaluación centrada en el resultado (10). Esos códigos sirvieron para caracterizar el funcionamiento general del trabajo en equipo experimentado por los estudiantes a partir de las prácticas realizadas.

Mediante la generación de códigos de atributo (Miles et al., 2014; Saldaña, 2009) “estudiantes CAFyD” y "estudiantes MEF”, se pudo comparar la información obtenida a partir de la titulación cursada. En la comparativa resultante (tabla 3 ), se observa que no todos los códigos que describen la dinámica de trabajo en equipo están presentes en la vivencia de cada grupo. A continuación, se profundiza en el análisis de ambos discursos, atendiendo a los códigos que los conforman y sus relaciones.

Tabla 3. Comparativa de códigos entre los dos grupos, CAFyD y MEF, sobre el funcionamiento de las dinámicas de trabajo en equipo experimentadas durante la formación cursada.

\begin{tabular}{l|l}
\hline CAFyD & MEPEF \\
\hline Formación (6) & Formación (10) \\
Procedimiento (7) & Procedimiento (9) \\
Prevalencia de contenido (5) & Prevalencia de contenido (6) \\
Conflicto de responsabilidades (4) & Conflicto de responsabilidades (8) \\
Monitoreo del profesorado (6) & $\begin{array}{l}\text { Evaluación centrada en } \\
\text { resultado (10) } \\
\text { Evaluación demandada (7) }\end{array}$ \\
& Monitoreo del profesorado (8) \\
& Evaluación demandada (8) \\
\hline
\end{tabular}

Como se observa, para el grupo de los estudiantes CAFyD existe una sutil menor carga de códigos que estructuran y caracterizan las experiencias de trabajo grupal en su formación. Estos estudiantes no consideran haber recibido un modelo formativo de referencia para aprender a trabajar en equipo.

La propia tradición y cadencia de trabajar en grupo, como metodología habitual en su formación, les permite abordar las tareas encomendadas en las disciplinas cursadas; por tanto, desarrollan un modelo autodidacta al enfrentarse a dichas tareas desde la óptica del trabajo grupal.

En general, los alumnos aprenden a trabajar en equipo porque después de cuatro años haciendo tra- bajos grupales, ya conoces a tus compañeros, has visto diferentes situaciones, has reconocido si te has equivocado alguna vez o deberías haberlo reconocido, desde mi punto de vista (3:28. Estudiante CAFyD).

La ausencia de un modelo formativo explícito de referencia explica un procedimiento estratégico para el abordaje de la tarea en común, que se inicia con el reparto de las cargas de trabajo entre los miembros del grupo. Los estudiantes CAFyD reconocen de base que el sistema de reparto simple de cargas no es equitativo $y$, por tanto, no se considera el realizado como un trabajo verdaderamente en equipo.

Nosotros, ahora que estamos en cuarto, no estamos sabiendo hacer trabajo en grupo porque va uno y se pone a hacerlo todo y otros nada y, entonces, el trabajo concreto está siendo de todo menos grupal (3:25. Estudiante CAFyD).

Este procedimiento o dinámica de trabajo grupal que describen se relaciona con una fuerte prevalencia del contenido a elaborar, frente al procedimiento de desarrollo de la competencia para realizar la tarea. De esta forma, el reparto de las cargas para abordar dicha tarea finaliza con el trabajo realizado por cada uno, uniéndolas en forma de producto final.

En la semana antes ya se nos come el tiempo, tú haces esto, tú esto y tú esto, y verdaderamente no hay trabajo de grupo. Así va muy separado, unos hacen una cosa, otro otra y otro otra, y al final uno lo ha juntado todo (3:40. Estudiante CAFyD).

Igualmente, el procedimiento de trabajo grupal experimentado genera ciertos conflictos de responsabilidades entre sus miembros, que parece se ocultan a fin de no afectar al resultado o producto final y a la armonía de la convivencia, pero no se resuelven, sino más bien persisten: "hay cosas que son más individuales... pero lo del trabajo en equipo es una tapadera tras otra... y ese es el problema" (3:78. Estudiante CAFyD). Este aspecto hace que se demande un perfil docente que asuma tareas de supervisión y control sobre el proceso de las dinámicas grupales, dentro de un modelo de evaluación procesual, integrada en el propio desarrollo del aprendizaje.

Sería muy buena idea al hacer un trabajo del que te han puesto la fecha, por ejemplo, para dentro de un mes, que el profesor recogiera lo que cada grupo y alumno ha realizado cada semana. (...) con ello, el profesor se está dando cuenta de quién está traba- 
jando y también los del propio grupo. Esto sería una buena manera de poder llevar un control (3:22. Estudiante CAFyD).

Para el caso de los estudiantes MEF, la formación para aprender a trabajar en equipo, también autogenerada (no enseñada), se fundamenta en la iniciativa de base para establecer unas normas con las que organizarse como grupo, para compartir y realizar conjuntamente una tarea: "a trabajar en grupo nadie te enseña, tú formas un grupo y con ellos estableces tus normas de funcionamiento" (3:107. Estudiante MEF). Esta ausencia de formación previa condiciona la elección de un procedimiento para desarrollar el trabajo grupal dentro de estas dinámicas, repartiendo las cargas de trabajo entre sus miembros, aun sabiendo que el sistema genera desequilibrios, problemas y roces relacionales; pero asumiendo el procedimiento establecido, sosteniendo que la comunicación y el respeto podrían ayudar en el desempeño.

Es simple, quedamos hoy y tú te encargas de esto, de esto y de esto, y siempre va a haber gente que tire más, es normal, ya que unos tienen más dotes de líder, otros que aceptan todo, y otros que se llevan bien. Con ello, al final se van solucionando los problemas gracias a poder hablar desde el respeto (3:32. Estudiante MEF).

Este planteamiento procedimental se relaciona con una prevalencia del contenido a desarrollar por encima del proceso, lo cual se integra en un modelo de evaluación más centrada en los resultados y no tanto en el proceso en sí de trabajo en equipo: "yo considero que no se evalúa el trabajo en grupo, solo se evalúa el resultado del trabajo en grupo" (3:34. Estudiante MEF).

El problema es que si tú vas a un profesor y le dices mira tengo este problema y no podemos trabajar juntos, lo primero que te dice es: ¡uff!, pues a estas alturas en qué grupo te vas a poner; ya están todos los trabajos muy avanzados. Ese yo creo que es el problema, que a través del trabajo en grupo quieren evaluar los contenidos. Entonces, al final, el trabajo en grupo no lo están teniendo en cuenta desde mi punto de vista (3:42.D1.MEF).

Además, esta forma de proceder desencadena conflictos de responsabilidades que se relacionan con la falta de compromiso para asumir las tareas en los tiempos previstos y la falta de honestidad, no afrontando el resto de los miembros la problemática generada y ocultándola. Finalmente, las experiencias vivenciadas revelan un sentido negativo, derrotista y de hartazgo sobre el método de trabajo grupal aplicado. De esta forma, se termina dando preferencia al individualismo frente a la colaboración, debido a los problemas generados y no resueltos. Para este grupo de estudiantes el peso y la relevancia de este código es muy pronunciada: "si ya partes de un grupo con una persona irresponsable, por mucho trabajo en grupo o en equipo que quieras hacer, es imposible" (3:20. Estudiante $\mathrm{MEF})$.

Es muy sangrante muchas veces el tapar a los compañeros; (...) bueno, si no has hecho tal tarea, no vas a decirlo. Al final, el profesor no sabe que este no ha hecho esto o que no ha ido a las reuniones de grupo. En asignaturas como expresión corporal teníamos que reflejar las reuniones que habíamos tenido, quién había faltado y la gente no es honesta, y tú muchas veces, si quieres ser honesto, al final vas a acabar mal con los compañeros y el trabajo en grupo ya no va a salir bien (3:24. Estudiante MEF).

Entonces qué es trabajo en grupo, ¿ser un pringado?, ¿trabajar el doble que los demás? Y si no, yo no lo veo de otra manera. A mí es una de las cosas que más me ha molestado en la carrera, el tema del trabajo en grupo: cuando trabajas con gente trabajadora, genial y perfecto (3:41. Estudiante MEF).

Finalmente, desde la experiencia del trabajo grupal se demanda un perfil de profesorado que monitorice, oriente, acompañe y se preocupe por el proceso y las situaciones de roce y conflicto generado. Además, se demanda un modelo de evaluación más formativa y compartida, negociando, discutiendo la valoración sobre el trabajo realizado con el propio estudiante y el propio grupo, generando un feedback verdaderamente formativo que les ayude.

Yo creo que se debería consensuar la nota, que se preocupe el profesor, que esté más cerca; ¿y cómo os habéis organizado?, ¿qué habéis hecho?, ¿habéis quedado?, ¿Y por qué no lo habéis hecho así?, ¿Y por qué habéis decidido realizarlo así?, y yo qué sé, preguntar al grupo, al alumno (3:35. Estudiante $\mathrm{MEF}$ ).

Yo en el trabajo en grupo no te he visto colaborar, no te he visto cooperar, no te he visto implicado en el grupo, entonces creo que no es justo que tú tengas la misma nota que tu compañero que sí le he visto preocupado, que ha venido a las tutorías, que le he visto más implicado que tú, y hablarlo y ver si es verdad (3:39. Estudiante MEF). 


\section{Discusión}

Inicialmente se aprecia en las dos titulaciones que existe una tendencia en el profesorado a emplear en sus asignaturas dinámicas de trabajo en equipo para desarrollar los conocimientos teóricos y prácticos. Con ello, se evidencia la importancia que se da a trabajar juntos y en equipo en la formación inicial del profesorado, en consonancia con estudios similares (MorenoAndrés et al. 2010; Ní Chróinín \& O’Sullivan, 2014; Scallon, 2004).

A pesar de que se ha verificado un discurso similar en los dos grupos de estudiantes, respecto a un concepto y procedimiento común sobre el trabajo en equipo y desde las experiencias vivenciadas durante su formación, se aprecia en ellos la ausencia de las habilidades y capacidades necesarias para mejorar esta competencia. Posiblemente, esto se deba a carencias formativas para abordar el trabajo grupal en clave de cooperación, centrándose más en el producto final y en el contenido curricular, que en el propio funcionamiento de equipo (Moreno et al., 2007). Por tanto, la tarea se convierte más en un fin que en un medio, desde situaciones que no capacitan para trabajar en equipo (Velázquez, 2013).

En el modelo de trabajo grupal descrito se detallan carencias de carácter comunicativo y relacional, provocadas por el desequilibrio entre las cargas y tareas asumidas (efecto polizón) y las capacidades puestas al servicio del equipo. Esto supone abandonos y renuncias en las funciones aceptadas, así como incapacidad para solucionar los problemas dados. Por tanto, el modelo autodidacta que aplica el alumnado le ha llevado a la conflictividad, al individualismo y a la competitividad en mayor medida que a la colaboración, tal y como describen Cuadrado et al. (2012), Del Canto et al. (2009) y Jhen y Mannix (2017). Por último, aun valorando positivamente la disposición prosocial de los estudiantes para afrontar los retos mediante dinámicas de equipo, se requiere que el docente realice un seguimiento de la cooperación que se necesita (Ros, 2006; Asún et al., 2019).

Aunque los docentes reconocen la importancia que, para la enseñanza superior, tienen los procesos metodológicos de trabajo grupal (Moreno-Andrés et al., 2010), después no aplican un modelo formativo suficientemente estructurado y secuenciado con el que atender todos los aspectos esenciales del trabajo en equipo, lo cual es percibido por el propio alumnado. A partir de Johnson y Johnson (1999) se propone un modelo pedagógico cooperativo que facilite la reflexión sobre el proceso de trabajo en equipo, la perseverancia en la responsabilidad individual, la ayuda mutua, la comunicación, las habilidades sociales y la vivencia del "nosotros" frente al "yo" (interdependencia positiva) de una forma mucho más significativa y profunda.

Este modelo cooperativo podría acercar, con mayor certeza, a un estilo de trabajo en equipo basado en el liderazgo compartido, el seguimiento mutuo de la tarea, el comportamiento de apoyo, la adaptación y la vivencia del esfuerzo individual en beneficio del equipo (Salas et al., 2005). Con ello se presenta a los estudiantes una idea de trabajo en equipo mucho más próxima a lo valorado por los profesionales en contexto (Cannon-Bowers et al., 1995; Chen et al., 2004; Leach et al., 2005; Villardón-Gallego, 2015). Además, coherentemente, ambos grupos de estudiantes demandan un modelo de evaluación formativa dirigido más al proceso que al resultado, que les permita orientar mejor la adquisición y desarrollo de competencias (Barba et al., 2020; Fraile et al., 2013; Pérez-Pueyo et al., 2020), desde estrategias como la autoevaluación y la coevaluación que ayuden al desarrollo de los modelos pedagógicos cooperativos (Herrero at al., 2020; Méndez-Giménez \& Fernández-Río, 2013; Velázquez et al., 2014). De esta manera, se pretende contribuir al desarrollo de la capacidad de reflexión sobre el pensamiento de equipo, la comunicación interpersonal y la confianza mutua. Estos aspectos pueden favorecer el liderazgo de equipo, la movilización de comportamientos prosociales de ayuda y, en definitiva, la orientación de las personas hacia el bien del equipo, tal y como consideran Salas et al. (2005) para la creación de equipos de trabajo.

Por último, con el propósito de promover la eficacia del trabajo en equipo, se considera necesario estructurar las experiencias de trabajo grupal mediante estrategias que permitan poner en acción habilidades interpersonales como la comunicación, la negociación o la adaptabilidad, según indica Velázquez (2013) en su pedagogía de la cooperación.

\section{Conclusiones}

Se considera necesario revisar el rol del profesorado como facilitador de los procesos cooperativos asegurándose de que, más allá de los resultados obtenidos por los equipos, se atiendan, analicen, adquieran y desarrollen en la propia acción los condicionantes técnicos para alcanzar la colaboración efectiva. La actividad docente debe partir de dinámicas cooperativas que permitan tomar conciencia de los aspectos necesarios para aprender a trabajar en equipo: el liderazgo compartido, el seguimiento mutuo de la tarea, el comportamiento de apoyo, la adaptabilidad y la 
orientación como equipo, junto con la comunicación interactiva constante. También, la evaluación formativa del proceso cooperativo precisa de estrategias por ejemplo de autoevalución y coevaluación, desde un proceso de aprendizaje interiorizado y debatido, garantizando un feedback que permita comparar la propia percepción con el intercambio de impresiones fruto de la coevaluación sistemática y continuada. La evaluación en la actividad cooperativa es fundamental ya que debe posibilitar que tras cada sesión puesta en marcha los participantes puedan examinar y verbalizar las conductas acaecidas. Durante el proceso el docente debe buscar soluciones para avanzar, fomentando y adoptando actitudes de equipo. La conducta de cooperación debe ayudar a superar las situaciones de conflicto y asegurar el desarrollo de la propia acción a través de habilidades más prosociales. Finalmente, se considera limitante en el estudio la exclusividad de la información proveniente de los estudiantes, siendo necesario complementar esta con otra procedente de experiencias e iniciativas de los docentes. Con ello, se trata de establecer una comparativa sobre el desarrollo del trabajo en equipo en el aula universitaria desde las dos perspectivas. Del mismo modo, se insta al uso de otros instrumentos de recogida de información como la entrevista en profundidad, con objeto de producir un conocimiento nuevo y valioso, que ayude a los futuros docentes a trabajar en equipo. Por todo ello, se deben optimizar los modelos formativos competenciales de formación del profesorado.

\section{Agradecimientos}

Este estudio forma parte del proyecto $\mathrm{I}+\mathrm{D}+\mathrm{i}$ : "Las competencias docentes en la formación inicial del profesorado de educación física", convocatoria de noviembre de 2013 del Programa Estatal de Investigación, Desarrollo e Innovación Orientada a los Retos de la Sociedad, en el marco del Plan Estatal de Investigación Científica y Técnica y de Innovación 2013-2016. Referencia: EDU 2013-42024-R.

\section{BIBLIOGRAFÍA}

Aguilera, A. (2018). ¿Cómo valoran los estudiantes de magisterio la docencia que reciben? Revista Fuentes, 20(1), 57-76. doi:10.12795/revistafuentes.2018.v20.i1.04.

Asún, S., Rapún, M., \& Romero, M. R. (2019). Percepciones de Estudiantes Universitarios sobre una Evaluación Formativa en el Trabajo en Equipo. Revista Iberoamericana de Evaluación Educativa, 12(1), 175192. doi: 10.15366/riee2019.12.1.010.

Barba-Martín, R., Bores-García, D., González-Calvo, G., \& HortigüelaAlcalá, D. (2020). Evaluación formativa con los estudiantes en prácticas, para reducir la brecha teoría-práctica en la formación inicial del profesorado. Educación Física y Deporte, 39(1). doi:10.17533/udea. efyd.v39n1a02.

Barbour, R. (2014). Los grupos de discusión en la investigación cualitativa. Madrid: Narcea.

Barbour, R., \& Kitzinger, J. (1999). Developing Focus Group Research: Politics, Theory and Practice. Londres: Sage.

Bloor, M., Frank, J., Thomas, M., \& Robson, K. (2001). Focus Groups in Social Research. Londres: Sage.

Cano, E., \& Fernández, M. (2016). Evaluación por competencias: La perspectiva de las primeras promociones de graduados en el EEES. Barcelona: Octaedro.

Cannon-Bowers, J. A., Tannenbaum, S. I., Salas, E., \& Volpe, C. E. (1995). Defining competencies and establishing team training requirements. In A. Guzzo y E. Salas (Eds.), Team effectiveness and decision making in organizations (pp. 259-265). San Francisco (CA): Jossey-Bass.

Casey, A., \& Dyson, B. (2009). The implementation of models-based practice in Physical Education through action research. European Physical Education review, 15(2), 175-199. doi:10-1177/1356336X09345222.

Cuadrado, C., Fernández-López, F., Fernández-López, M., FernándezPacheco, C. González, D., \& Lifante, I. (2012). Técnicas de trabajo en equipo para estudiantes universitarios. In X Jornades de Xarxes d'Investigació en Docència Universitària: la participació i el compromís de la comunitat universitària (pp. 3072-3086). Alicante: Instituto de Ciencias de la Educación.

Chen, G., Donahue, L. M., \& Klimoski, R. J. (2004). Training undergraduates to work in organizational teams. Academy of Management Learning and Education, 3(1), 27-40. doi: 10.5465/amle.2004.12436817.
Del Canto, P., Gallego, I., López, J. M., Mora, J., Reyes, A., Rodríguez, E., Sanjeevan, K., Santamaría, E., \& Valero, M. (2009). Conflictos en el trabajo en grupo: cuatro casos habituales. Revista de Formación e Innovación Educativa Universitaria, 2(4), 211-226.

Fossey, E., Harvey, C., McDermott, F., \& Davidson, L. (2002). Understanding and evaluating qualitative research. Australian and New Zealand Journal of Psychiatry, 36(6), 717-732. doi:10.1046/j.14401614.2002.01100.x.

Flick, U. (2007). Introducción a la investigación cualitativa. Londres: Sage. Fraile, A. (2008). El aprendizaje cooperativo como metodología para el desarrollo de los ECTS: una experiencia de formación del profesorado de educación física. Fuentes: Revista de la Facultad de Ciencias de la Educación, 8, 22-35. doi:10.12795/revistafuentes.

Fraile, A., Juez, A., \& Julián, J. A. (2009). Estrategias de aprendizaje cooperativo mediante un proyecto de acción grupal: análisis de una experiencia universitaria de educación física. Tándem, 31, 82-92.

Fraile, A., López-Pastor, V., Castejón, F. J., \& Romero, M. R. (2013). La evaluación formativa en docencia universitaria y el rendimiento académico del alumnado. Aula Abierta, 41(2), 22-34. doi:10.17811/ rifie.41.1-3.2013.

Fernández-Río, J. (2017). El Ciclo del Aprendizaje Cooperativo: una guía para implementar de manera efectiva el aprendizaje cooperativo en educación física. Retos, 32, 244-249.

Ferriz-Valero, A., García-Martínez, S., \& Arroyo-Botella, J. M. (2019). Metodología cooperativa disminuye las actitudes disruptivas en educación física. Revista Internacional de Medicina y Ciencias de la Actividad Física y del Deporte, 19(76), 599-615. doi:10.15366/rimcafd2019.76.

Gallego-Ortega, J. L., \& Rodríguez-Fuentes, A. (2018). Percepciones del profesorado sobre competencias comunicativas de futuros maestros de educación física. Revista Internacional de Medicina y Ciencias de la Actividad Física y del Deporte, 18(71), 479-492. doi:10.15366/rimcafd2018.71.

Gámez, M. J., \& Torres, C. (2013). Group techniques as a methodological strategy in acquiring teamwork abilities by college students. Journal for Educators, Teachers and Trainers, 4(2), 14-25.

Gibbs, G. (2012). El análisis de datos cualitativos en Investigación Cualitativa. Madrid: Narcea. 
Guba, E. (1985). Criterios de credibilidad en la investigación naturalista. In J. Gimeno Sacristán y A. Pérez Gómez (Eds.), La enseñanza: Su teoría y su práctica (pp. 148-166). Madrid: Akal.

Herrero, D., López-Pastor, V., \& Manrique, J. C. (2020). La evaluación formativa y compartida en contextos de Aprendizaje Cooperativo en Educación Física en Primaria. Revista Ciencia, Cultura y Deporte, 15(44), 213-222. doi:10.12800/ccd.v15i44.1463.

Howe, R. (2011). Assessing Team work. College Documentation Bulletin, 7, 1-12.

Jhen, K. A., \& Mannix, E. A. (2017). The dynamic nature of conflict: A longitudinal study of intragroup conflict and group performance. Academy of Management Journal, 44(2), 238-251. doi:10.5465/3069453.

Johnson, D. W., \& Johnson, R. T. (1999). Aprender juntos y solos. Aprendizaje cooperativo, competitivo e individualista. Buenos Aires: Aique.

Kozlowski, S., \& Ilgen, D. (2006). Enhancing the effectiveness of work groups and teams. Psychological Science in the Public Interest, 7(3), 77 124. doi:10.1111/j.1529-1006.2006.00030.x.

Leach, D. J., Wall, T. D., Rogelberg, S. G., \& Jackson, P. R. (2005). Team autonomy, performance and member job strain: Uncovering the teamwork KSA link. Applied Psychology: An International Review, 54(1) 1-24. doi:10.1111/j.1464-0597.2005.00193.x.

López-Pastor, V., \& Pérez-Pueyo, A. (coords.) (2017). Evaluación formativa y compartida en Educación: experiencias de éxito en todas las etapas educativas. León: Universidad de León.

Méndez-Alonso, D., Méndez-Giménez, A., \& Fernández-Río, J. (2016). Incorporación de las competencias básicas a la educación física en educación Primaria. Revista Internacional de Medicina y Ciencias de la Actividad Física y del Deporte, 16(63), 457-473. doi:10.15366/rimcafd2016.63.

Méndez-Giménez, A., \& Fernández-Río, J. (2013). El aprendizaje cooperativo en la formación del profesorado: una experiencia basada en autoconstrucción de materiales e invención de juegos. Revista Española de Educación Física y Deporte, 400, 55-78.

Miles, M., Huberman, M., \& Saldaña, J. (2014). Qualitative Data Analysis: A Methods Sourcebook. Londres: Sage.

Molina, J. P., \& Antolín, L. (2008). Las competencias básicas en educación física: una valoración crítica. Cultura, Ciencia y Deporte, 3, 81-86. doi:10.12800/ccd.v3i8.200.

Moreno-Andrés, M. V., Quesada-Pallarés, C., \& Pineda-Herrero, P. (2010). El grupo de trabajo como método innovador de formación del profesorado para potenciar la transferencia del aprendizaje. Revista Española de Pedagogía, 246, 281-295.

Moreno, S., Bajo, M. T., Moya, M., Maldonado, A., \& Tudela, P. (2007) Las competencias en el nuevo paradigma educativo para Europa. Granada: Universidad de Granada.

Ní Chróinín, D., \& O’Sullivan, M. (2014). From initial teacher education through induction and beyond: A longitudinal study of primary teacher beliefs. Irish Educational Studies, 33, 451-466. doi:10.1080/033 23315.2014.984387.

Pérez Pueyo, A., Hortigüela-Alcalá, D., \& Fernández-Río, J. (2020). Evaluación formativa y modelos pedagógicos: estilo actitudinal, aprendizaje cooperativo, modelo comprensivo y educación deportiva. Revista Española de Educación Física y Deportes, 428, 47-66.

Pérez-Pueyo, A., Hortigüela-Alcalá, D., \& Hernando, A. (2013). La coevaluación intragrupal y el reparto de notas bajo un proceso de evaluación formativa. In P. Membiela, N. Casado y M. I. Cebreiros, Experiencias e innovación docente en el contexto actual de la docencia universitaria (pp. 285-289). Orense: Educación Editora.

Prieto, J. R, Alarcón, D., \& Fernández, C. B. (2018). Aprendizaje y evaluación de competencias en el universitario de ciencias sociales. REDU: Revista de Docencia Universitaria, 16(1), 193-210. doi:10.4995/ redu.2018.8941.
Ros, J. A. (2006). Análisis de roles de trabajo en equipo: un enfoque centrado en comportamientos (Tesis Doctoral). Barcelona: Universidad Autónoma de Barcelona.

Sáiz, M. I., \& Gómez, G. R. (2011). Aprendizaje autónomo y trabajo en equipo: reflexiones desde la competencia percibida por los estudiantes universitarios Revista Electrónica Interuniversitaria de Formación del Profesorado, 14(2), 73-85.

Salas, E., Sims, D. E., \& Burke, C. S. (2005). Is there a" big five" in teamwork? Small Group Research, 36(5), 555-599.

Saldaña, J. (2009). The Coding Manual for Qualitative Researchers. Thousand Oaks, CA: Sage.

Sundstrom, E., Demeuse, K. P., \& Futrell, D. (1999). Work Teams: Applications and effectiveness. American Psychologist, 45(2), 120-123. doi:10.1037/0003-066X.45.2.120.

Scallon, G. (2004). L'évaluation des apprentissages dans une approche par compétences. Saint-Laurent: Éditions du Renouveau Pédagogique.

Strauss, A., \& Corbin, C. (1998). Basics of qualitative research. Techniques and procedures for developing grounded theory. Londres: Sage.

Torrelles, C., Coiduras, J., Isus, S., Carrera, F. X., París, G., \& Cela, J. M. (2011). Competencia de trabajo en equipo: Definición y categorización. Profesorado. Revista de Currículum y Formación del Profesorado, 15(3), 329-344

Torrelles, C., Coiduras Rodríguez, J., Carrera Farran, X., \& Isus, S. (2014). Du changement dans l'évaluaction du travail d'équipe: Validation de l'outil RUTE. Les Dossiers de Sciences de l'Éducation, 31, 101-116. doi:10.4000/dse.603.

Torrelles, C., Paris, G., Sabriá, B., \& Alsinet, C. (2015). Assessing teamwork competence. Psicothema, 27(4), 354-361. doi:10.7334/psicothema2014.284.

Vallejo-Ruiz, M., \& Molina-Saorín, J. (2011). Análisis de las metodologías activas en el grado de maestro en educación infantil: La perspectiva del alumnado. Revista Electrónica Interuniversitaria de Formación del Profesorado, 14(1), 207-217.

Velázquez, C. (2013). La pedagogía de la cooperación en educación física. Armenia: Kínesis.

Velázquez, C. (2018). El enfoque de coopedagogía como pieza clave en la transformación social. In E. Lorente y D. Martos (eds.), Educación Física y pedagogía crítica. Propuestas para la transformación personal y social, (pp. 273-291). Lleida: Universitat de Lleida.

Velázquez, C., Fraile, A., \& López-Pastor, V. (2014). Aprendizaje cooperativo en Educación Física. Movimento, 20(1), 239-259. doi:10.22456 1982-8918.40518.

Villa, A., \& Poblete, M. (2007). Aprendizaje basado en competencias: Una propuesta para la evaluación de las competencias genéricas: Bilbao: Mensajero.

Villardón-Gallego, L. (2015). Competencias Genéricas en Educación Superior: Metodologías específicas para su desarrollo. Madrid: Narcea.

Watling Neal, J., Neal, Z., VanDyke, E., \& Kornbluh, M. (2015). Expediting the Analysis of Qualitative Data in Evaluation: A Procedure for the Rapid Identification of Themes from Audio Recordings (RITA). American Journal of Evaluation, 36(1), 118-132. doi:10.1177/ 1098214014536601.

Wolf, N. H., \& Silver, C. (2017). Qualitative analysis using ATLAS.ti. New York: Routledge.

Yilmaz, K. (2013). Comparison of quantitative and qualitative research traditions: Epistemological, theoretical, and methodological differences. European Journal of Education, 48(2), 311-325. doi:10.1111/ ejed.12014.

Zapatero, J. A., González, M. D., \& Campos, A. (2018). El modelo competencial en Educación Física: contribución, evaluación y vinculación con sus contenidos. Cultura, Ciencia y Deporte, 3, 17-30. doi:10.12800/ ccd.v13i37.1035. 